\title{
Introducing Switching Ordered Statistic CFAR Type I in Different Radar Environments
}

\author{
Saeed Erfanian and Vahid Tabataba Vakili \\ Electrical Engineering Department, Iran University of Science \& Technology (IUST), Narmak, Tehran 16846, Iran \\ Correspondence should be addressed to Saeed Erfanian, s_erfanian@iust.ac.ir
}

Received 22 September 2008; Revised 3 December 2008; Accepted 17 February 2009

Recommended by M. Greco

\begin{abstract}
In this paper, a new CFAR detector based on a switching algorithm and OS-CFAR for nonhomogeneous background environments is introduced. The new detector is named Switching Ordered Statistic CFAR type I (SOS CFAR I ). The SOS CFAR I selects a set of suitable cells and then with the help of the ordering method, estimates the unknown background noise level. The proposed detector does not require any prior information about the background environment and uses cells with similar statistical specifications to estimate the background noise. The performance of SOS CFAR I is evaluated and compared with other detectors such as CA-CFAR, GO-CFAR, SO-CFAR, and OS-CFAR for the Swerling I target model in homogeneous and nonhomogeneous noise environments such as those with multiple interference and clutter edges. The results show that SOS CFAR I detectors considerably reduce the problem of excessive false alarm probability near clutter edges while maintaining good performance in other environments. Also, simulation results confirm the achievement of an optimum detection threshold in homogenous and nonhomogeneous radar environments by the mentioned processor.
\end{abstract}

Copyright (c) 2009 S. Erfanian and V. Tabataba Vakili. This is an open access article distributed under the Creative Commons Attribution License, which permits unrestricted use, distribution, and reproduction in any medium, provided the original work is properly cited.

\section{Introduction}

A common routine test in any detection system is to compare the received signal level with a predefined threshold value. If the threshold is crossed, the presence of the signal of interest is declared. In modern radar detection, the decision on target presence or absence is often performed automatically, that is, without the visual intervention of the radar operator. When the threshold is a fixed value, the false alarm rate will increase intolerably (i.e., beyond a level that the computer of an automatic detector can handle) as the interference power varies. In this situation, a constant false alarm rate (CFAR) algorithm with an adaptive threshold is required to keep the false alarm rate constant.

In a radar receiver, after amplitude detection, the backscattered signal is sampled in range and/or Doppler and a one- or two-dimensional reference window is formed. The detection in radar means existence or nonexistence of a target in the middle cell of a reference window or a cell under test (CUT). The noise and clutter background is estimated by processing the output from neighbouring cells. A well- known group for noise estimation is mean-level detectors such as cell averaging CFAR (CA) [1]. Unfortunately because of differences in environmental conditions such as change in clutter edge, multiple targets, or jamming the target detection will be corrupted. As solutions for these problems, various CFAR schemes are proposed. A few examples are the greatest of CFAR (GO-CFAR), the smallest of CFAR (SO-CFAR), order statistics CFAR (OS-CFAR), the excision cell-averaging CFAR (EXCA-CFAR), and the excision of the greatest of CFAR (EXGO-CFAR) [2, 3]. Each of these schemes has advantages and disadvantages but none of them shows considerably good performance in all types of environments. However, the processors which use ordering have better performance than mean levels.

Following up on the results in $[4,5]$ we focus on a new type of switching processor which we call Switching Ordered Statistic CFAR type I (SOS CFAR I). SOS I can be used in many instances, especially in nonhomogeneous environments, and in this paper its performance will be analysed in comparison with conventional CFAR processors in the presence of clutter edge and multiple targets. The suggested 
detector is based on comparing cells with scaled CUT to set the cells with the same statistical specifications in two groups. By counting the number of cells in each group and finding the group with more cells, estimation of background noise will be performed [4]. In this paper after describing the SOS CFAR I algorithm in Section 2, mathematical and related probabilities of detection and false alarms are presented in Section 3. In Section 4 the performance and simulation of the SOS I processor in homogeneous and nonhomogeneous environments will be analysed, and in the last section the results are presented.

\section{Description of SOS CFAR I Method}

In this paper, it is assumed that the CFAR processor's inputs are range samples (range cells) which are received from the square law detector and are saved into a tapped delay line of length $2 N+1$. The $2 N$ samples correspond to reference cells $X_{i}$ surrounding the test cell $X_{0}$. The SOS I detector block diagram is provided in Figure 1. Here, single pulse detection and a Rayleigh fading model are assumed for fluctuating targets corresponding to Swerling I in single pulse processing. For a homogeneous noise pulse clutter level, the in-phase I and quadrature Q input signals are assumed independent and identically distributed (iid) Gaussian random variables with zero mean. Consequently, the output samples of the square law detector are also iid R.V.s with an exponential distribution [6,7]. Thus, the probability density function (PDF) of the $i$ th cell is

$$
f_{X_{i}}\left(x_{i}\right)=\frac{1}{\lambda} e^{-x_{i} / \lambda}, \quad x_{i} \geq 0, \lambda \geq 0,1 \leq i \leq 2 N
$$

in which $X_{i}$ s are $2 N$ window samples (excluding the CUT), and $\lambda$ is the total background clutter-plus-thermal noise power. If a cell contains thermal noise then $\lambda=\lambda_{0}=2 \eta$, and if it consists of clutter then $\lambda=\lambda_{c}=2 \eta\left(1+\sigma_{c}\right)$. If a cell consists of multiple (not primary) targets then in (2) $\lambda=\lambda_{I}=2 \eta\left(1+\sigma_{I}\right)$. Also $\sigma_{c}$ is the ratio of clutter's power to the noise power, and $\sigma_{I}$ is the ratio of multiple targets' power to the noise.

Target detection in CUT is carried out by estimating the $2 N$ reference window cells that surround it. The PDF of CUT is the same as (1) in the case of thermal noise with $\lambda=\lambda_{0}=$ $2 \eta$, and in the case of primary (main) target it will be in the form of (2) with $\lambda=\lambda_{s}=2 \eta\left(1+\sigma_{s}\right)$ while $\sigma_{s}$ is the ratio of the signal power to the noise power:

$$
f_{X_{0}}\left(x_{0}\right)=\frac{1}{\lambda} e^{-x_{0} / \lambda}, \quad x_{0} \geq 0, \lambda \geq 0 .
$$

In Figure 1 the SOS I detector first divides reference samples into two $S_{1}$ and $S_{0}$ groups by comparing them with scaled $X_{0}$ with $\alpha<1$. This is a criterion for finding samples with the same specification. In other words, it implies collecting samples with the same amplitude in one group. Next, estimation of the background noise will be done based on $S_{0}$ group or $2 N$ samples of reference window. The manner of selection is based on comparing the number of $S_{0}$ group samples $\left(n_{0}\right)$ with an integer threshold $N_{T}$. If $n_{0}$ is more

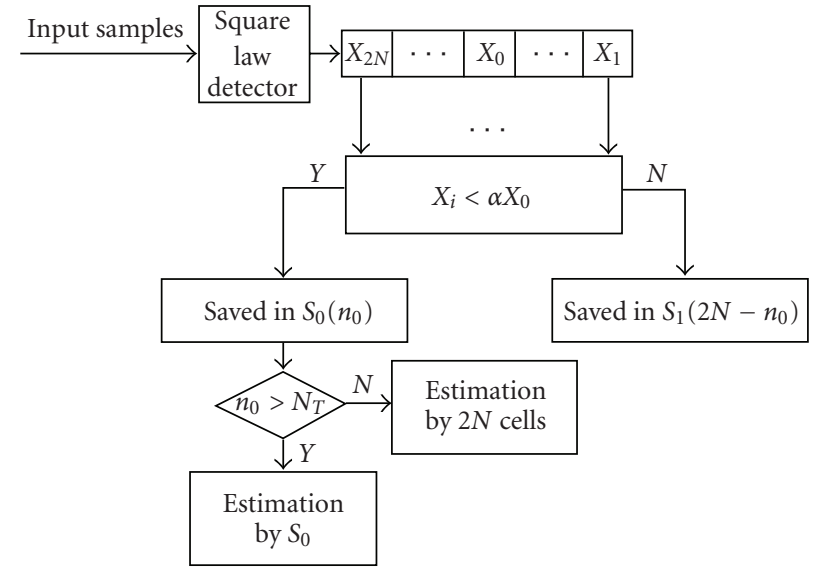

FIgURE 1: Block diagram of SOS CFAR I.

than $N_{T}$, background noise will be estimated only with the $S_{0}$ samples, but if $n_{0}$ is less than $N_{T}$, similar to SCFAR $[8,9]$, all the samples of the reference window are selected. In both cases, background noise estimation is obtained from one of the ordered samples of $S_{0}$ or the whole reference window. The range samples in both groups are first ordered according to their magnitudes, and the estimation is taken to be the $k$ th largest sample. Also, $N_{T}$ is selected based on detector requirements and environment conditions. This process gives the detector an ability to suppress the masking effect caused by interfering targets and clutter edge. Thus, the algorithm will be carried out in the following two steps.

(i) $2 \mathrm{~N}$ cells in the reference window will be compared with the scaled CUT by $\alpha(\alpha<1)$. If a cell value is less than $\alpha X_{0}$ it will be saved in group $S_{0}$, otherwise it will be saved in $S_{1}$ as in

$$
X_{i} \stackrel{S_{S_{0}}}{\geq} \alpha X_{0}, \quad i=1,2, \ldots, 2 N .
$$

(ii) If the number of samples saved in group $S_{0}$ is $n_{0}$, then the target will exist in CUT according to the following conditions:

$$
\text { If } X_{0}>\beta_{0} X_{\left(k_{0}\right)}=\beta_{0} Z_{0}, \quad \text { when } n_{0}>N_{T} \text {, }
$$

or

$$
\text { If } X_{0}>\beta_{1} X_{\left(k_{1}\right)}=\beta_{1} Z_{1} \text {, when } n_{0} \leq N_{T} \text {, }
$$

where $\beta_{0}$ and $\beta_{1}$ are constants for achieving the desired false alarm probability, and $N_{T}$ is the threshold integer. Also, in (4) and (5) $k_{0}$ and $k_{1}$ are rounded $\left(g_{0} \times n_{0}\right)$ and $\left(g_{1} \times 2 N\right)$ where $g_{0}$ and $g_{1}$ are parameters between 0 and 1 . By adjusting $g_{0}$ and $g_{1}$, the order of each selected group is achieved.

Inequalities (4) and (5) mean that SOS CFAR I switches between the sample set $S_{0}$ and whole reference, depending on the value of $n_{0}$. For example, if the number of samples which have a value lower than the scaled CUT (and are saved in $S_{0}$ ) is more than the preset threshold $N_{T}$, noise level estimation is carried out by ordering the homogeneous saved samples in 
$S_{0}$ and selecting $k_{0}$ th of them; but if the number of samples which have a value lower than the scaled CUT is less than the considered threshold, the noise level estimation is carried out by ordering homogeneous saved samples in the whole reference window and selecting $k_{1}$ th of them. This type of processing by the SOS CFAR I processor means selecting an optimized threshold of detection in homogeneous and nonhomogeneous environments.

\section{Mathematical Analysis of SOS CFAR I}

Switching Ordered Statistics type I CFAR detector incorporates a switching method to estimate the total noise power. Such a detector is specifically tailored to provide good estimates of the noise power with an exponential PDF. In this section we analyse the performance of the SOS CFAR I processor in a homogeneous background as well as in regions of clutter transitions and in multiple target environments. We obtain closed-form performance expressions in each case.

3.1. Homogeneous Environment. Considering the algorithm described in Section 2 and considering the existence of $n_{0}$ samples in $S_{0}$ and $2 N-n_{0}$ samples in $S_{1}$, and by referring to (4) and (5), the detection probability of SOS CFAR I is

$$
P_{d}=P_{d_{0}}+P_{d_{1}}
$$

in which $P_{d_{0}}$ is the probability of detection when $S_{0}$ is selected, and $P_{d_{1}}$ is the probability of detection when the whole reference window is selected. If we assume presence of a target, $H_{1}$, we have

$P_{d}=P\left(\right.$ when $S_{0}$ is selected $\left.\mid H_{1}\right)$

$+P\left(\right.$ when the whole reference window is selected $\left.\mid H_{1}\right)$,

which is equal to

$P_{d}$

$=$ Probability of saving $\left(N_{T}+1 \leq n_{0} \leq 2 N\right)$ samples in $S_{0}$

$\times$ Probability that $X_{0}$ is more than $\left(\beta_{0} Z_{0}\right)$

+ Probability of saving $\left(0 \leq n_{0} \leq N_{T}\right)$ samples in $S_{0}$

$\times$ Probability that $X_{0}$ is more than $\left(\beta_{1} Z_{1}\right)$.

Therefore, the probability of detection when the whole reference window is selected is

$$
P_{d_{1}}=\sum_{n_{0}=0}^{N_{T}} P_{s} \times P\left(X_{0}>\beta_{1} Z_{1} \mid H_{1}\right)
$$

where $P_{s}$ is the probability that there are (based on (8)) exactly $n_{0}$ noise samples in $S_{0}$ and is equal to

$$
P_{S}=E_{X_{0}}\left\{\left(\begin{array}{c}
2 N \\
n_{0}
\end{array}\right) P_{0}^{n_{0}}\left(x_{0}\right)\left(1-P_{0}\left(x_{0}\right)\right)^{2 N-n_{0}}\right\} \text {. }
$$

$P_{0}\left(x_{0}\right)$ is the probability that a noise sample belongs to $S_{0}$, which is computed as

$$
\begin{aligned}
P_{0}\left(x_{0}\right) & =P\left(X_{i}<\alpha X_{0}\right) \\
& =\int_{x_{i}=0}^{\alpha x_{0}} f_{X_{i}}\left(x_{i}\right) d_{x_{i}} \\
& =\int_{x_{i}=0}^{\alpha x_{0}} \frac{1}{\lambda} e^{-x_{i} / \lambda} d_{x_{i}} \\
& =1-e^{-(\alpha / \lambda) x_{0}} .
\end{aligned}
$$

In (11), it is assumed that the samples are independent, the window samples contain thermal noise and the CUT contains signal [10]. By using (A.1) in Appendix A, (10) becomes

$$
P_{S}=\left(\begin{array}{c}
2 N \\
n_{0}
\end{array}\right) \sum_{i=0}^{n_{0}}\left(\begin{array}{c}
n_{0} \\
i
\end{array}\right) \frac{(-1)^{i}}{\alpha\left(2 N-n_{0}+i\right)\left(1+\sigma_{s}\right)+1}
$$

In (9), $Z_{1}$ is the random variable obtained from the ordering of $2 N$ noise samples in the reference window and selecting $k_{1}$ of them as an estimation of noise in the case of $n_{0}<N_{T}$. Now, if as (5) we consider $Z_{1}=X_{(k 1)}$, then the PDF of $Z_{1}$ is given by [11]

$$
f_{K_{1}}\left(z_{1}\right)=K_{1}\left(\begin{array}{c}
2 N \\
K_{1}
\end{array}\right)\left(e^{-z_{1} / \lambda}\right)^{2 N-K_{1}}\left(1-e^{-z_{1} / \lambda}\right)^{K_{1}-1} \frac{1}{\lambda} e^{-z_{1} / \lambda}
$$

Therefore by referring to Appendix B,

$$
\begin{aligned}
& P\left(X_{0}>\beta_{1} Z_{1} \mid H_{1}\right) \\
& =\frac{(2 N) !}{\left(2 N-k_{1}\right) !} \sum_{m=0}^{k_{1}-1} \frac{(-1)^{m}}{m !\left(k_{1}-m-1\right) !} \frac{1}{2 N-k_{1}+m+\beta_{1} /\left(1+\sigma_{s}\right)} .
\end{aligned}
$$

For calculating the probability of detection when only the samples in $S_{0}$ are selected, similar to (9), one has

$$
P_{d_{0}}=\sum_{n_{0}=N_{T}+1}^{2 N} P_{s} \times P\left(X_{0}>\beta_{0} Z_{0} \mid H_{1}\right)
$$

where $P_{S}$ was calculated in (10). Therefore same as (14),

$$
\begin{aligned}
& P\left(X_{0}>\beta_{0} Z_{0} \mid H_{1}\right) \\
& =\frac{n_{0} !}{\left(n_{0}-k_{0}\right) !} \sum_{m=0}^{k_{0}-1} \frac{(-1)^{m}}{m !\left(k_{0}-m-1\right) !} \frac{1}{n_{0}-k_{0}+m+\beta_{0} /\left(1+\sigma_{s}\right)} .
\end{aligned}
$$




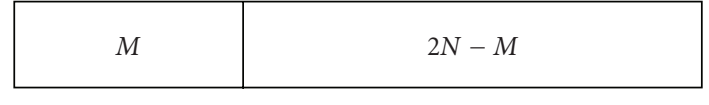

Whole reference

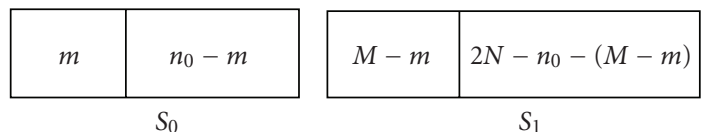

Figure 2: Position of noise samples in nonhomogeneous environment.

By using (9) and (15), the result will be

$P_{d}^{\mathrm{SOSI}}\left(N_{T}, \alpha, \beta_{0}, \beta_{1}, g_{0}, g_{1}\right)$

$=\sum_{n_{0}=0}^{N_{T}}\left(\begin{array}{c}2 N \\ n_{0}\end{array}\right) \sum_{i=0}^{n_{0}}\left(\begin{array}{c}n_{0} \\ i\end{array}\right) \frac{(-1)^{i}}{\alpha\left(2 N-n_{0}+i\right)\left(1+\sigma_{\mathrm{s}}\right)+1} \frac{(2 N) !}{\left(2 N-k_{1}\right) !}$

$\times \sum_{m=0}^{k_{1}-1} \frac{(-1)^{m}}{m !\left(k_{1}-m-1\right) !} \frac{1}{2 N-k_{1}+m+\beta_{1} /\left(1+\sigma_{s}\right)}$

$+\sum_{n_{0}=N_{T}+1}^{2 N}\left(\begin{array}{c}2 N \\ n_{0}\end{array}\right) \sum_{i=0}^{n_{0}}\left(\begin{array}{c}n_{0} \\ i\end{array}\right) \frac{(-1)^{i}}{\alpha\left(2 N-n_{0}+i\right)\left(1+\sigma_{\mathrm{s}}\right)+1} \frac{n_{0} !}{\left(n_{0}-k_{0}\right) !}$

$\times \sum_{m=0}^{k_{0}-1} \frac{(-1)^{m}}{m !\left(k_{0}-m-1\right) !} \frac{1}{n_{0}-k_{0}+m+\beta_{0} /\left(1+\sigma_{s}\right)}$.

Also, using (17) and setting $\sigma_{s}$ equal to zero, the probability of occurrence of a false alarm can be determined as

$$
\begin{aligned}
P_{\mathrm{fs}}^{\mathrm{SOS} I} & \left(N_{T}, \alpha, \beta_{0}, \beta_{1}, g_{0}, g_{1}\right) \\
= & \sum_{n_{0}=0}^{N_{T}}\left(\begin{array}{c}
2 N \\
n_{0}
\end{array}\right) \sum_{i=0}^{n_{0}}\left(\begin{array}{c}
n_{0} \\
i
\end{array}\right) \frac{(-1)^{i}}{\alpha\left(2 N-n_{0}+i\right)+1} \frac{(2 N) !}{\left(2 N-k_{1}\right) !} \\
& \times \sum_{m=0}^{k_{1}-1} \frac{(-1)^{m}}{m !\left(k_{1}-m-1\right) !} \frac{1}{2 N-k_{1}+m+\beta_{1}} \\
& +\sum_{n_{0}=N_{T}+1}^{2 N}\left(\begin{array}{c}
2 N \\
n_{0}
\end{array}\right) \sum_{i=0}^{n_{0}}\left(\begin{array}{c}
n_{0} \\
i
\end{array}\right) \frac{(-1)^{i}}{\alpha\left(2 N-n_{0}+i\right)+1} \frac{n_{0} !}{\left(n_{0}-k_{0}\right) !} \\
& \quad \times \sum_{m=0}^{k_{0}-1} \frac{(-1)^{m}}{m !\left(k_{0}-m-1\right) !} \frac{1}{n_{0}-k_{0}+m+\beta_{0}} .
\end{aligned}
$$

3.2. Nonhomogeneous Environment. Considering the algorithm described in Section 2, it is assumed that in a reference window with a size equal to $2 N$, there are $M$ interfering samples and $2 N-M$ thermal noise samples, and $S_{0}$ contains $m$ interfering samples and $n_{0}-m$ thermal noise samples as illustrated in Figure 2. Assuming that there are $n_{0}$ samples in $S_{0}$ and $2 N-n_{0}$ samples in $S_{1}$ and by referring to (4) and (5), the detection probability in SOS I will be as follows.
For $M$ interfering samples that appear in the CFAR window, the probability that exactly $m$ of them are stored in $S_{0}$ is

$$
q_{1 s}=\left(\begin{array}{c}
M \\
m
\end{array}\right)\left(P_{0}^{\prime}\left(X_{0}\right)\right)^{m}\left(1-P_{0}^{\prime}\left(X_{0}\right)\right)^{M-m} .
$$

For $2 N-M$ thermal noise samples that appear in the CFAR window, the probability that exactly $n_{0}-m$ of them are stored in $S_{0}$ is [8]

$$
q_{0 s}=\left(\begin{array}{c}
2 N-M \\
n_{0}-m
\end{array}\right)\left(P_{0}\left(X_{0}\right)\right)^{n_{0}-m}\left(1-P_{0}\left(X_{0}\right)\right)^{2 N-M-\left(n_{0}-m\right)} .
$$

Therefore, the probability that there are exactly $m$ interfering samples and $n_{0}-m$ thermal noise samples in $S_{0}$ is

$$
\begin{aligned}
Q_{s}=q_{0 s} \times q_{1 s} & \\
=E_{X_{0}}\left\{\sum_{m=m_{1}}^{\min \left(M, n_{0}\right)}\right. & \left(\begin{array}{c}
2 N-M \\
n_{0}-m
\end{array}\right)\left(\begin{array}{l}
M \\
m
\end{array}\right)\left(P_{0}\left(X_{0}\right)\right)^{n_{0}-m} \\
& \times\left(1-P_{0}\left(X_{0}\right)\right)^{2 N-M-\left(n_{0}-m\right)} \\
& \left.\times P_{0}^{\prime m}\left(X_{0}\right)\left(1-P_{0}^{\prime}\left(X_{0}\right)\right)^{M-m}\right\} .
\end{aligned}
$$

Here, the probability of existence of a sample with thermal noise in the $S_{0}$ group is determined by (11). Also, the probability of existence of a sample with interference noise in $S_{0}$ group according to (3) is

$$
\begin{aligned}
P_{0}^{\prime}\left(X_{0}\right) & =P\left(X_{i}<\alpha X_{0}\right) \\
& =\int_{x_{i}=0}^{\alpha x_{0}} f_{X_{i}}\left(X_{i}\right) d_{x_{i}} \\
& =\int_{x_{i}=0}^{\alpha x_{0}} \frac{1}{\lambda_{1}} e^{-x_{i} / \lambda_{I}} d_{x_{i}} \\
& =1-e^{-\left(\alpha / \lambda_{I}\right) x_{0}} .
\end{aligned}
$$

Therefore, with the help of (11) and (22) and referring to Appendix C, (21) will be

$$
\begin{aligned}
Q_{s}= & \sum_{m=m_{1}}^{\min \left(M, n_{0}\right)}\left(\begin{array}{c}
2 N-M \\
n_{0}-m
\end{array}\right)\left(\begin{array}{c}
M \\
m
\end{array}\right) \\
& \times \sum_{t=0}^{n_{0}-m} \sum_{q=0}^{m} \frac{(-1)^{t+q}\left(\begin{array}{c}
n_{0}-m \\
t
\end{array}\right)\left(\begin{array}{c}
m \\
q
\end{array}\right)}{1+\mathfrak{N}\left(1+\sigma_{s}\right) \alpha+(M-m+q)\left(\left(1+\sigma_{s}\right) /\left(1+\sigma_{I}\right)\right) \alpha},
\end{aligned}
$$

where $\mathfrak{N}$ denotes $\left(2 N-M-\left(n_{0}-m\right)+t\right)$. In the equation above, $m_{1}$ is equal to $\max \left(0, n_{0}-2 N+M\right)$. Now, similar to (6), 
the probability of detection in the case of interfering targets will be

$$
\begin{aligned}
P_{d}^{\text {SOS I }} & \left(N_{T}, \alpha, \beta_{0}, \beta_{1}, g_{0}, g_{1}\right) \\
= & \sum_{n_{0}=0}^{N_{T}} Q_{s} \times P\left(X_{0}>\beta_{1} Z_{1} \mid H_{1}\right) \\
& +\sum_{n_{0}=N_{T}+1}^{2 N} Q_{s} \times P\left(X_{0}>\beta_{0} Z_{0} \mid H_{1}\right) .
\end{aligned}
$$

Referring to Appendix D, in the case of a nonhomogeneous environment, one has

$$
\begin{aligned}
& P\left(X_{0}>\beta_{1} Z_{1} \mid H_{1}\right) \\
& =\frac{\beta_{1}}{\left(1+\sigma_{s}\right)} \sum_{i=k_{1}}^{2 N} \sum_{L=p_{1}}^{p_{2}}\left(\begin{array}{c}
2 N-M \\
L
\end{array}\right)\left(\begin{array}{c}
M \\
i-L
\end{array}\right) \\
& \quad \times \sum_{j_{1}=0}^{L} \sum_{j_{2}=0}^{i-L} \frac{\left(\begin{array}{c}
L \\
j_{1}
\end{array}\right)\left(\begin{array}{c}
i-L \\
j_{2}
\end{array}\right)(-1)^{j_{1}+j_{2}}}{2 N-M-L+\beta_{1} /\left(1+\sigma_{s}\right)+j_{1}+\mathfrak{Q} /\left(1+\sigma_{I}\right)},
\end{aligned}
$$

where $\mathfrak{Q}$ denotes $\left(j_{2}+M-i+L\right)$. And with the same procedure in the $S_{0}$ group,

$$
\begin{aligned}
& P\left(X_{0}>\beta_{0} Z_{0} \mid H_{1}\right) \\
& =\frac{\beta_{0}}{\left(1+\sigma_{s}\right)} \sum_{i=k_{0}}^{2 N} \sum_{L=t_{1}}^{t_{2}}\left(\begin{array}{c}
n_{0}-m \\
L
\end{array}\right)\left(\begin{array}{c}
m \\
i-L
\end{array}\right) \\
& \quad \times \sum_{j_{1}=0}^{L} \sum_{j_{2}=0}^{i-L} \frac{\left(\begin{array}{c}
L \\
j_{1}
\end{array}\right)\left(\begin{array}{c}
i-L \\
j_{2}
\end{array}\right)(-1)^{j_{1}+j_{2}}}{n_{0}-m-L+\beta_{0} /\left(1+\sigma_{s}\right)+j_{1}+\mathfrak{C} /\left(1+\sigma_{I}\right)},
\end{aligned}
$$

where $\mathfrak{C}$ denotes $\left(j_{2}+m-i+L\right)$ and $t_{1}$ and $t_{2}$ are equal to $\max (0, i-m)$ and $\min \left(i, n_{0}-m\right)$.

Now we investigate the performance of the SOS I processor when the reference window contains a clutter edge. First, consider the special case where CUT is not from the clutter region. Also similar to the multiple targets case, it is assumed that in a reference window with a size equal to $2 \mathrm{~N}$, there are $M$ samples from clutter and $2 N-M$ thermal noise samples, and $S_{0}$ contains $m$ samples from clutter and $n_{0}-m$ thermal noise samples. First, the probability of existence of a sample with thermal noise in the $S_{0}$ group according to (3) was calculated in (11). Also, the probability of existence of a sample with clutter noise in the $S_{0}$ group according to (22) is

$$
\begin{aligned}
P_{0}^{\prime}\left(X_{0}\right) & =P\left(X_{i}<\alpha X_{0}\right) \\
& =\int_{x_{i}=0}^{\alpha x_{0}} f_{X_{i}}\left(x_{i}\right) d_{x_{i}} \\
& =\int_{x_{i}=0}^{\alpha x_{0}} \frac{1}{\lambda_{C}} e^{-x_{i} / \lambda_{C}} d_{x_{i}} \\
& =1-e^{-\left(\alpha / \lambda_{C}\right) x_{0}} .
\end{aligned}
$$

Here with the help of (23), (24), (25), and (26) and considering $\sigma_{s} \rightarrow 0$ and $\sigma_{I} \rightarrow \sigma_{C}$, the $P_{f a}^{\text {SOS I }}$ will be

$$
\begin{aligned}
P_{f a}^{\mathrm{SOSI}}( & \left.N_{T}, \alpha, \beta_{0}, \beta_{1}, g_{0}, g_{1}\right) \\
= & \sum_{n_{0}=0}^{N_{T}} Q_{s} \times P\left(X_{0}>\beta_{1} Z_{1} \mid H_{0}\right) \\
& +\sum_{n_{0}=N_{T}+1}^{2 N} Q_{s} \times P\left(X_{0}>\beta_{0} Z_{0} \mid H_{0}\right),
\end{aligned}
$$

where

$$
\begin{aligned}
& P\left(X_{0}>\beta_{1} Z_{1} \mid H_{0}\right) \\
& =\beta_{1} \sum_{i=k_{1}}^{2 N} \sum_{L=p_{1}}^{p_{2}}\left(\begin{array}{c}
2 N-M \\
L
\end{array}\right)\left(\begin{array}{c}
M \\
i-L
\end{array}\right) \\
& \quad \times \sum_{j_{1}=0}^{L} \sum_{j_{2}=0}^{i-L} \frac{\left(\begin{array}{c}
L \\
j_{1}
\end{array}\right)\left(\begin{array}{c}
i-L \\
j_{2}
\end{array}\right)(-1)^{j_{1}+j_{2}}}{2 N-M-L+\beta_{1}+j_{1}+\mathfrak{Q} /\left(1+\sigma_{C}\right)}, \\
& P\left(X_{0}>\beta_{0} Z_{0} \mid H_{0}\right) \\
& =\beta_{0} \sum_{i=k_{0}}^{2 N} \sum_{L=t_{1}}^{t_{2}}\left(\begin{array}{c}
n_{0}-m \\
L
\end{array}\right)\left(\begin{array}{c}
m \\
i-L
\end{array}\right) \\
& \quad \times \sum_{j_{1}=0}^{L} \sum_{j_{2}=0}^{i-L} \frac{\left(\begin{array}{c}
L \\
j_{1}
\end{array}\right)\left(\begin{array}{c}
i-L \\
j_{2}
\end{array}\right)(-1)^{j_{1}+j_{2}}}{n_{0}-m-L+\beta_{0}+j_{1}+\mathfrak{C} /\left(1+\sigma_{C}\right)},
\end{aligned}
$$

where

$$
\begin{aligned}
Q_{s}= & \sum_{m=m_{1}}^{\min \left(M, n_{0}\right)}\left(\begin{array}{c}
2 N-M \\
n_{0}-m
\end{array}\right)\left(\begin{array}{c}
M \\
m
\end{array}\right) \\
& \times \sum_{t=0}^{n_{0}-m} \sum_{q=0}^{m} \frac{(-1)^{t+q}\left(\begin{array}{c}
n_{0}-m \\
t
\end{array}\right)\left(\begin{array}{c}
m \\
q
\end{array}\right)}{1+\mathfrak{N} \alpha+(M-m+q)\left(1 /\left(1+\sigma_{C}\right)\right) \alpha} .
\end{aligned}
$$

Now if CUT is from the clutter region, after substituting $\sigma_{I}=$ $\sigma_{s}, \beta_{0}, \beta_{1}$, and $\alpha$ by $\sigma_{C}, \beta_{0} /\left(1+\sigma_{c}\right), \beta_{1} /\left(1+\sigma_{c}\right)$, and $\alpha\left(1+\sigma_{c}\right)$ in (23), (24), (25), (26), and also

$$
\begin{aligned}
& P\left(X_{0}>\beta_{1} Z_{1} \mid H_{0}\right) \\
& =\frac{\beta_{1}}{1+\sigma_{C}} \sum_{i=k_{1}}^{2 N} \sum_{L=p_{1}}^{p_{2}}\left(\begin{array}{c}
2 N-M \\
L
\end{array}\right)\left(\begin{array}{c}
M \\
i-L
\end{array}\right) \\
& \quad \times \sum_{j_{1}=0}^{L} \sum_{j_{2}=0}^{i-L} \frac{\left(\begin{array}{c}
L \\
j_{1}
\end{array}\right)\left(\begin{array}{c}
i-L \\
j_{2}
\end{array}\right)(-1)^{j_{1}+j_{2}}}{2 N-M-L+\beta_{1} /\left(1+\sigma_{C}\right)+j_{1}+\mathfrak{Q} /\left(1+\sigma_{C}\right)} . \\
& P\left(X_{0}>\beta_{0} Z_{0} \mid H_{0}\right) \\
& =\frac{\beta_{0}}{1+\sigma_{C}} \sum_{i=k_{0}}^{2 N} \sum_{L=t_{1}}^{t_{2}}\left(\begin{array}{c}
n_{0}-m \\
L
\end{array}\right)\left(\begin{array}{c}
m \\
i-L
\end{array}\right) \\
& \quad \times \sum_{j_{1}=0}^{L} \sum_{j_{2}=0}^{i-L} \frac{\left(\begin{array}{c}
L \\
j_{1}
\end{array}\right)\left(\begin{array}{c}
i-L \\
j_{2}
\end{array}\right)(-1)^{j_{1}+j_{2}}}{n_{0}-m-L+\beta_{0} /\left(1+\sigma_{C}\right)+j_{1}+\mathfrak{C} /\left(1+\sigma_{C}\right)},
\end{aligned}
$$




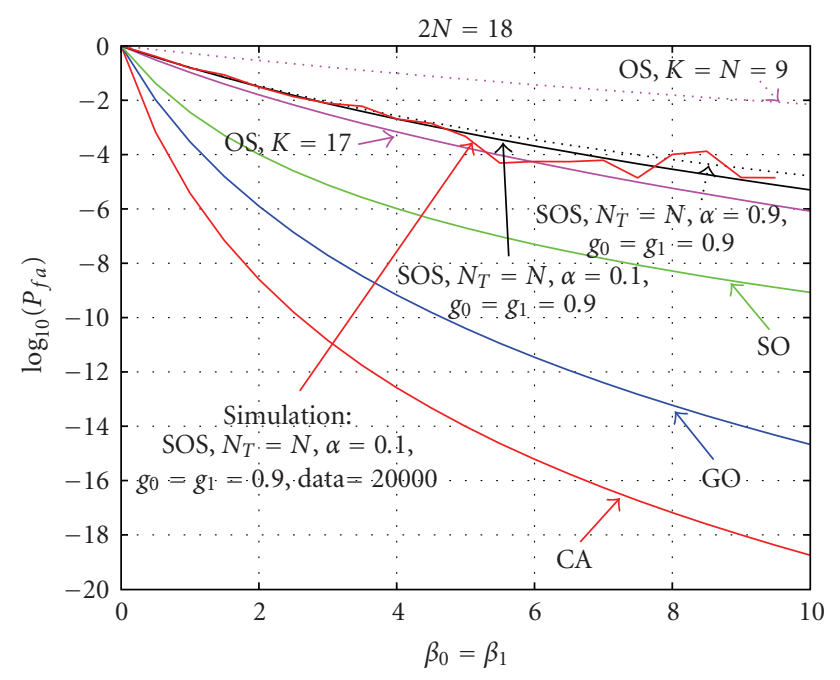

FIgUre 3: False alarm probability of the CA, GO, SO, OS $(k=N=9$ and $k=17)$, and SOS CFAR I processors for $2 N=18$.

where

$$
\begin{aligned}
Q_{s}=\sum_{m=m_{1}}^{\min \left(M, n_{0}\right)}\left(\begin{array}{c}
2 N-M \\
n_{0}-m
\end{array}\right)\left(\begin{array}{l}
M \\
m
\end{array}\right) \\
\\
\times \sum_{t=0}^{n_{0}-m} \sum_{q=0}^{m} \frac{(-1)^{t+q}\left(\begin{array}{c}
n_{0}-m \\
t
\end{array}\right)\left(\begin{array}{c}
m \\
q
\end{array}\right)}{1+\mathfrak{N}\left(1+\sigma_{C}\right) \alpha+(M-m+q) \alpha} .
\end{aligned}
$$

\section{Studying SOS CFAR I in Different Conditions}

The performance of the Switching Ordered Statistic CFAR I processor algorithm, according to (11), is a function of $\beta_{0}, \beta_{1}, N_{T}, \alpha, g_{0}$, and $g_{1}$. These parameters should be tuned such that the SOS CFAR I processor has minimum CFAR loss when operating in a homogeneous environment. Provided that there are only noise samples within the CFAR window, almost all reference samples will be stored to $S_{0}$ if the test cell contains a target return signal with substantial SNR. The SOS CFAR I processor then tends to switch to $S_{0}$, and the threshold multiplier $\beta_{0}$ and $g_{0}$ are employed with high probability. In order to minimise the CFAR loss in this situation, $g_{0}\left(k_{0}\right)$ should be set as close as possible to the order of OS CFAR (corresponding to the false alarm probability of interest). If the test cell contains no target signal, far fewer reference samples are sorted to $S_{0}$. The whole CFAR window and ordering $g_{1}\left(k_{1}\right)$ are then employed with high probability. In order to maintain the false alarm rate as that of the OSCFAR, $g_{1}\left(k_{1}\right)$ should also be set as close as possible to $k$ of the OS. Therefore, a reasonable choice is $g_{0}=g_{1}$. Also, for preventing complexity, $\beta_{0}=\beta_{1}$ is considered, although different values for $\beta_{0}$ and $\beta_{1}$ could be considered for the future. The setting of the SOS CFAR I parameters for the case $g_{0}=g_{1}$ and $\beta_{0}=\beta_{1}$ are briefly discussed in this section [8].

In order to detect targets near a clutter edge, the threshold integer should be approximately equal to or smaller than

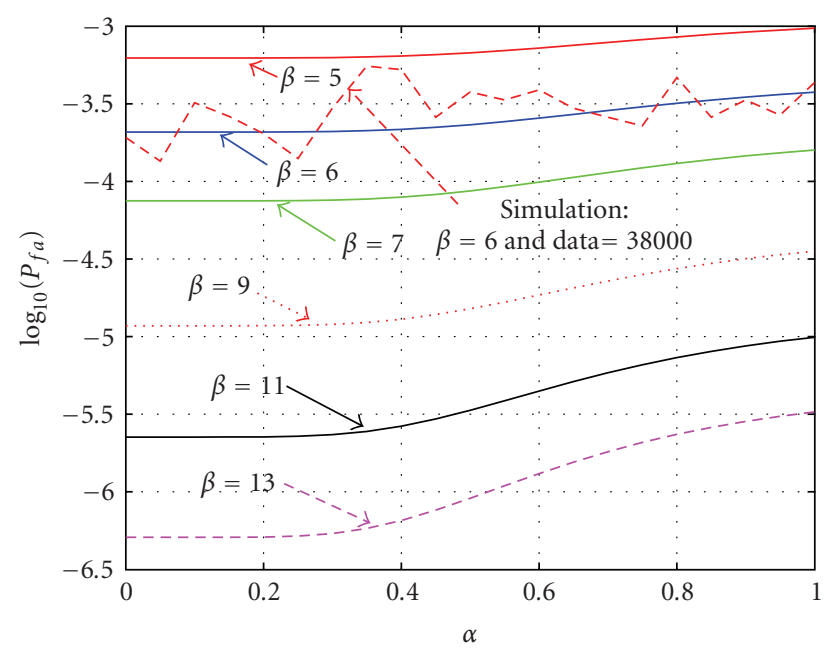

FIGURE 4: False alarm probability of the SOS CFAR I processor for different $\beta_{0}=\beta_{1}$ in terms of $\alpha$.

the half window size, that is, $N_{T} \leq N$. An SOS CFAR I processor with a smaller $N_{T}$ can tolerate a greater number of interference samples, but in a homogeneous environment it suffers from more CFAR loss compared to the CACFAR. Here CFAR loss, based on $[12,13]$, is defined as the additional SNR the CFAR processor requires in order to achieve the same detection probability at a given false alarm rate.

The curves in Figure 3 are the false alarm probabilities $\left(P_{f a}\right)$ for a reference window with the size $2 N=18$ for SOS I, with $N_{T}=N=9, \beta_{0}=\beta_{1}, g_{0}=g_{1}=0.9, \alpha=0.1$, and $\alpha=$ $0.9, \mathrm{CA}, \mathrm{GO}, \mathrm{SO}$, and OS (with $k=N=9$ and $k=17$ ) in a homogeneous environment. It is seen that in a homogeneous environment, only $P_{f a}$ of OS with $k=N=9$ is worse than SOS I. For verifying the theoretical results, the performance of SOS I with $N_{T}=N=9, \beta_{0}=\beta_{1}, g_{0}=g_{1}=0.9$, and $\alpha=0.1$ has been simulated by the Monte Carlo method for about 20000 data for each point. As it is shown, this curve is compatible with the analytical curve.

In Figure 4, $P_{f a}$ of the performance of SOS I processor in a homogeneous environment with $2 N=18, N_{T}=N$, and $g_{0}=g_{1}=0.9$ have been plotted for $\alpha$ and different values of $\beta_{0}=\beta_{1}$. It is clear that by increasing $\beta_{0}=$ $\beta_{1}, P_{f a}$ is decreasing. Also, it is seen that in all cases with $\alpha$ larger than 0.4 and with $\beta_{0}=\beta_{1}, P_{f a}$ is increasing. Also in Figure 4 the curve for $\beta=6$ has been simulated with the Monte Carlo method for about 38000 data for each point which is compatible with the analytical curve with the same parameters.

In Figure 5, $P_{f a}$ of the SOS I processor in a homogeneous environment with $2 N=18, \beta_{0}=\beta_{1}=9$, and $\alpha=0.5$ and for different $N_{T}$ values have been plotted for $g_{0}=g_{1}$. It is clear that by increasing $N_{T}, P_{f a}$ is decreasing.

The probability of the occurrence of a false alarm by the SOS I detector in a homogeneous environment based on different $N_{T}$ and $\alpha$ values have been plotted for $g_{0}=g_{1}$ in Figure 6; here $\beta_{0}=\beta_{1}=9$. It is seen that by decreasing $N_{T}$ 


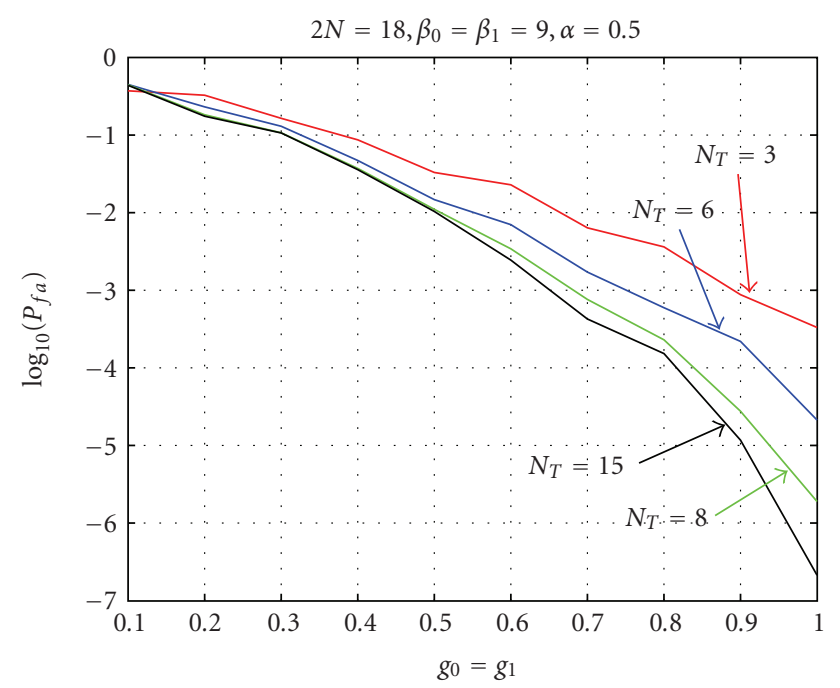

FIgURe 5: Comparison of $P_{f a}$ in SOS I with different $N_{T}$ in terms of $g_{0}=g_{1}$ in homogeneous environment with $2 N=18$.

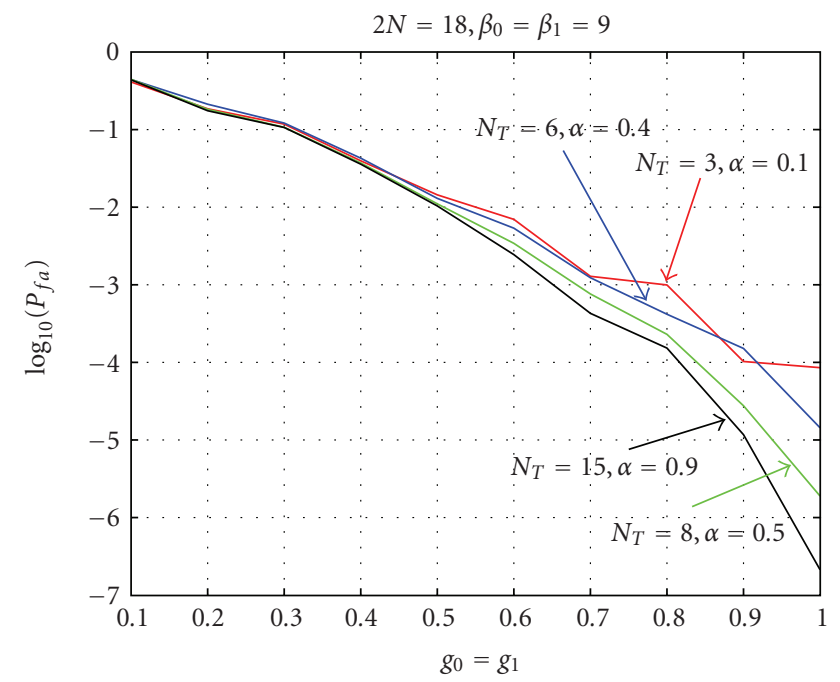

FIgURE 6: Comparison of $P_{f a}$ for SOS I proce $\alpha$ sor in homogeneous environment in terms of $g_{0}=g_{1}$ and some different $N_{T}$ and $\alpha$ with $2 N=18$.

and increasing $\alpha, P_{f a}$ is decreasing. Also it is clear that by increasing both $N_{T}$ and $\alpha$ parameters, $P_{f a}$ is again decreasing.

Now, in Figure 7, the detection probability of the SOS I detector in a homogeneous environment in comparison with the optimum detector, $\mathrm{CA}, \mathrm{GO}, \mathrm{SO}$, and OS (with $k=N=9$ and $k=17$ ) and for $P_{f a}=10^{-5}$, has been drawn. The optimum detector sets a fixed threshold to determine the presence of a target under the assumption that the total homogeneous noise power is known a priori [10]. Considering the loss detection, it is seen that the SOS I processor with $N_{T}=N, \alpha=0.1, \beta_{0}=\beta_{1}=9$, and $g_{0}=g_{1}=0.9$ has inherent detection loss in the homogeneous environment which is more than CA and GO but is less than SO and OS $(k=N=9)$. Also, this figure shows that by increasing the order of OS to $k=17$, its detection loss will be

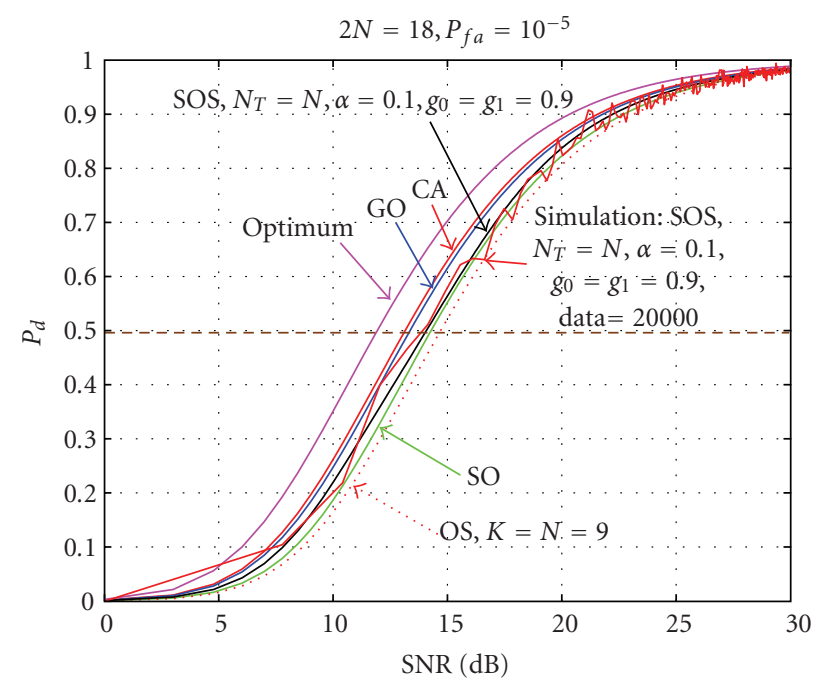

Figure 7: Comparison of $P_{d}$ for CA, GO, SO, OS $(k=N=9$ and $k=17)$, and SOS I processors $\left(2 N=18\right.$ and $\left.P_{f a}=10^{-5}\right)$.

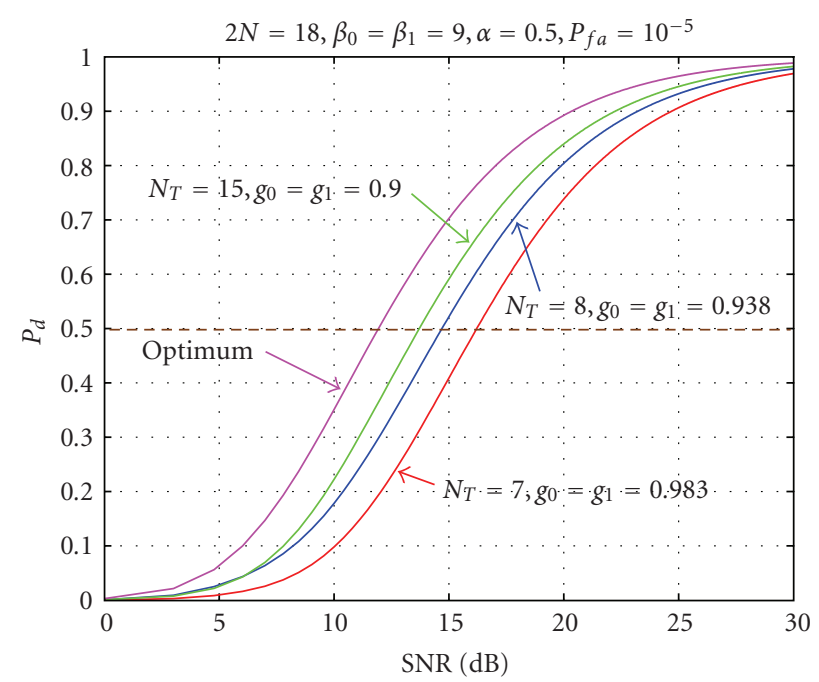

FIgure 8: Comparison of $P_{d}$ for SOS I with different $N_{T}$ and $g_{0}=g_{1}$ $\left(2 N=18\right.$ and $\left.P_{f a}=10^{-5}\right)$.

less than the SOS I detector with the mentioned parameters. In fact, with the help of Figure 3, increasing $k$ in OS causes less detection loss but a higher probability of false alarm. For better comparison, the $P_{d}$ of SOS I is achieved by the Monte Carlo simulation with 20000 data for each point. As Figure 7 shows, the result of the Monte Carlo simulation is the same as the analysis result of Section 3.

In Figure 8 the detection probability of the SOS I detector in a homogeneous environment with different values of $N_{T}, g_{0}=g_{1}$ and for $P_{f a}=10^{-5}, \beta_{0}=\beta_{1}=9$, and $\alpha=0.5$ has been plotted. The result shows that with greater $N_{T}$ and smaller $g_{0}=g_{1}$, it has less detection loss.

Next, the performance of the SOS I in presence of clutter edge is analysed. The result in Figure 9 is achieved in the presence of clutter edge with clutter to noise ratio (CNR) 


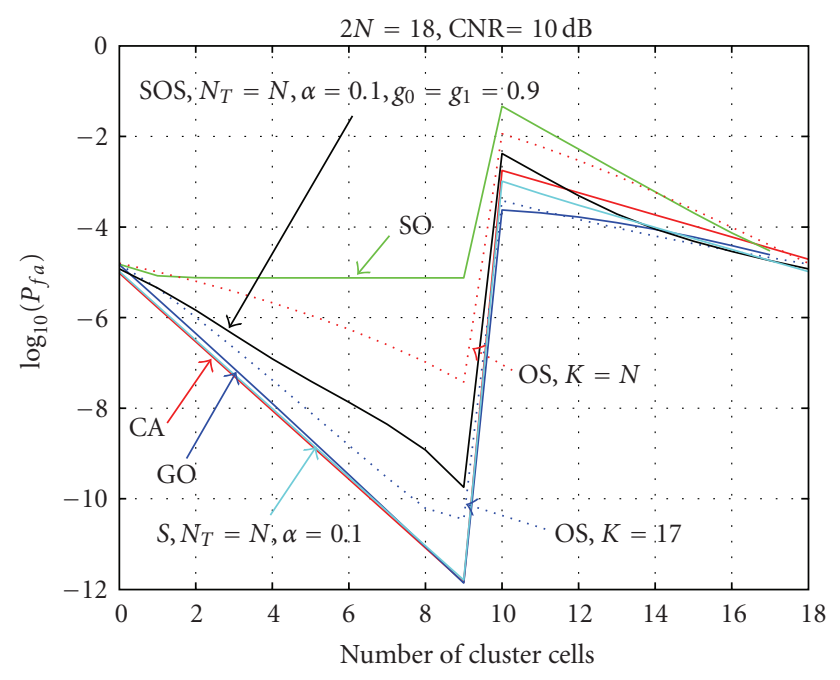

Figure 9: Comparison of $P_{f a}$ for CA, GO, SO, OS $(k=N=9)$, and SOS I processors $(2 \mathrm{~N}=18$ and $\mathrm{CNR}=10 \mathrm{~dB})$.

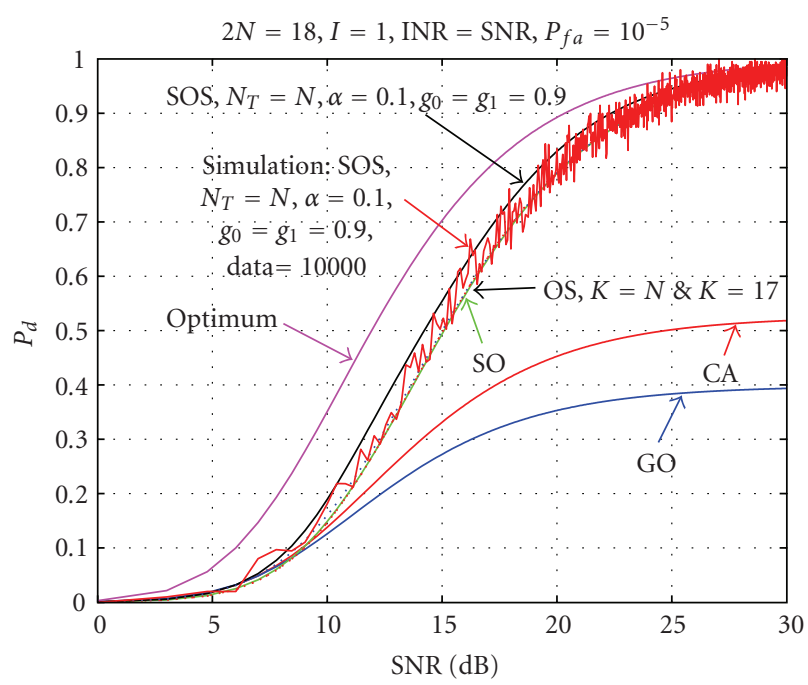

Figure 10: Comparison of $P_{d}$ of SOS I by CA, GO, SO, and OS $(k=N$ and $k=17)$ in the case of one multiple targets (INR $=\mathrm{SNR})$ for $P_{f a}=10^{-5}$ and $2 N=18$.

equal to $10 \mathrm{~dB}, P_{f a}=10^{-5}$, and $2 \mathrm{~N}=18$. It is known that in the presence of clutter edge, the GO processor has the lowest probability of false alarm and is followed by CA, $S$ with $N_{T}=N, \alpha=0.1$, OS $(k=17)$, and SOS I with $N_{T}=N, \alpha=0.1$, and $g_{0}=g_{1}=0.9$. It is clear from Figure 9 that OS $(k=N=9)$ and SO are after it, and also GO has the best performance among all the CFAR processors in the presence of clutter.

The presence of multiple targets is another case in studying the SOS I processor. In Figure 10 one interfering target with interference to noise ratio (INR) equal to SNR and the size of reference window $2 \mathrm{~N}=18$ for CA, GO, SO, OS ( $k=N=9$ and $k=17)$, and SOS I processors

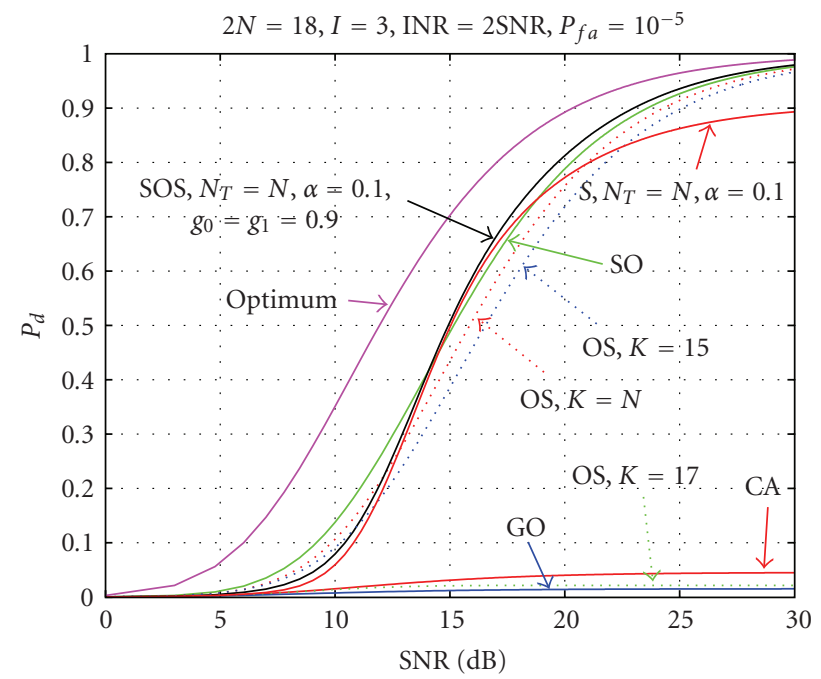

Figure 11: Comparison of $P_{d}$ of SOS I by CA, GO, SO, and OS ( $k=N=9, k=15$, and $k=17)$ in the case of three multiple targets $(\mathrm{INR}=2 \mathrm{SNR})$ for $P_{f a}=10^{-5}$ and $2 \mathrm{~N}=18$.

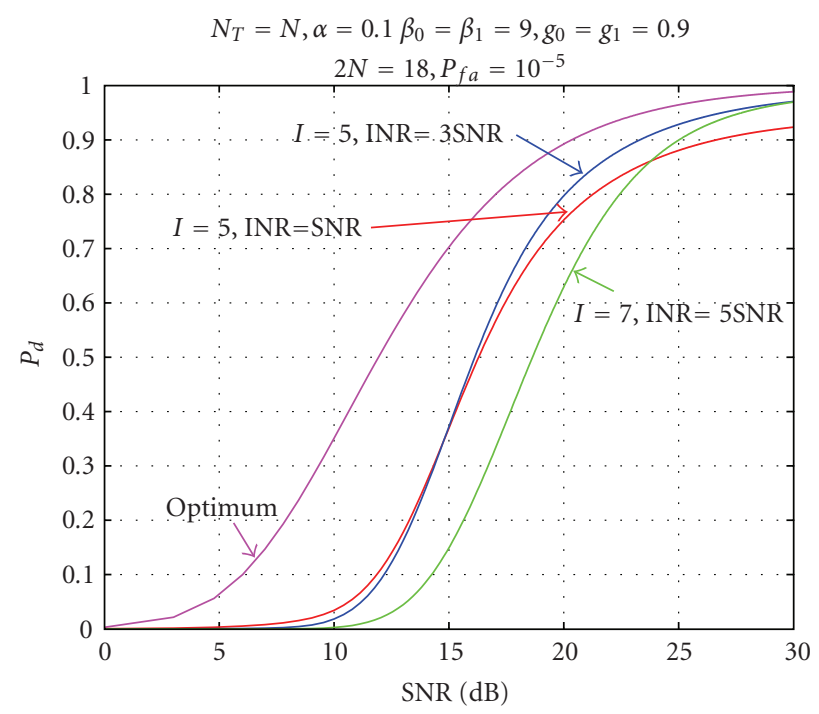

Figure 12: Comparison of $P_{d}$ of SOS I with $N_{T}=N, \alpha=0.1, \beta_{0}=$ $\beta_{1}=9$, and $g_{0}=g_{1}=0.9$ in multiple targets environment for $2 N=18$ and $P_{f a}=10^{-6}$.

with considered parameter in this figure and for $P_{f a}=10^{-5}$ are considered. As the result shows, SOS I has the best performance. By increasing the order of OS, its performance will become constant and will be equal to $\mathrm{SO}$ which have less $P_{d}$ in terms of SOS I with considered parameters. The result of the Monte Carlo simulation for 10000 data for each point is also confirmed by the result of theoretical analysis. It is noticeable that if data numbers for each point increase, the Monte Carlo simulation will have better compatibility with theoretical results.

If there is more than one multiple target, for example, 3 targets with INR $=2 \mathrm{SNR}$, Figure 11 can be considered. The other conditions of this figure are the same as the previous 


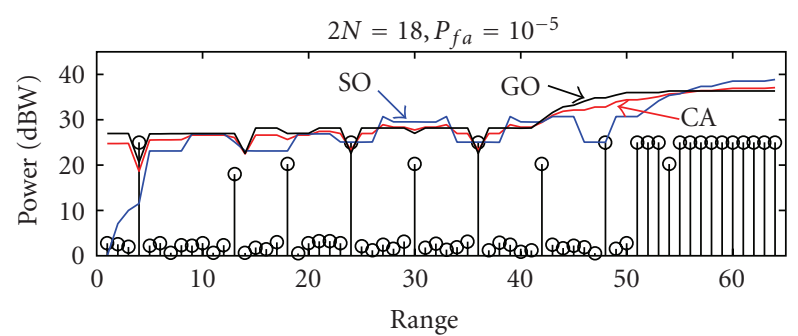

(a)

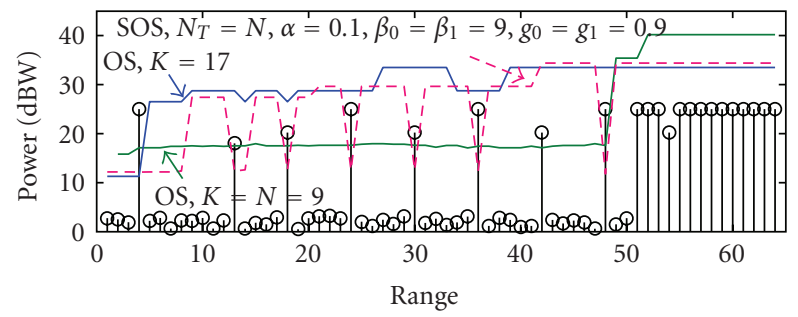

(b)

Figure 13: Detection thresholds of CA, GO, SO, OS $(k=N=9$ and $17)$, and SOS I $\left(N_{T}=N, \alpha=0.1, \beta_{0}=\beta_{1}=9\right.$, and $\left.g_{0}=g_{1}=0.9\right)$ in worse case $\left(2 N=18\right.$ and $\left.P_{f a}=10^{-5}\right)$.

figure. The results show that SOS I has the best performance and following it, there is the SO detector and then OS with $k=N=9$. If $k$ increases (e.g., $k=15$ and $k=17$ are considered), its performance decreases and even will be worse than the CA detector. Also, Figure 11 shows that the $P_{d}$ of $S$ with $N_{T}=N$ and $\alpha=0.1$ in the case of three multiple targets and INR $=2 \mathrm{SNR}$ will be reduced, and its performance is less than SOS I and SO with on SNR of more than $16 \mathrm{~dB}$.

In Figure $12, P_{d}$ for the case of five and seven multiple targets and different INR values is shown. The results show that $P_{d}$ of this detector for $I=5$ and INR $=3 \mathrm{SNR}$ is the highest. If in this case INR decreases, then $P_{d}$ for an SNR higher than $16 \mathrm{~dB}$ will decrease. $I=7$ and INR $=5$ SNR have the lowest $P_{d}$. In this figure, $2 N=18, N_{T}=N, \alpha=0.5, \beta_{0}=$ $\beta_{1}=9, g_{0}=g_{1}=0.9$, and $P_{f a}=10^{-5}$.

The detection threshold simulation is carried out using Matlab software in the presence of clutter and multiple targets. In Figures 13(a)-13(b), there are 8 targets in ranges $4,13,18,24,30,36,42$, and 48 with the SNR values mentioned in the figure. Considering the cases with the reference window's sizes equal to $2 \mathrm{~N}=18$ and $P_{f a}=10^{-5}$ and from Figure 13(a), the CA processor can only detect the first target while GO can detect the 1st, 4th, and 6th targets and SO can detect the 1st, 4th, 6th, and 8th targets. From Figure 13(b), OS ( $k=N=9)$ can detect all the targets, OS $(k=17)$ detects only the 1 st, and SOS with the mentioned parameters detects all the targets except the 7th target.

In Figure 14 the effect of changing SOS I parameters on its detection threshold has been analysed. As seen, SOS I with $\beta_{0}=\beta_{1}=149$ has the worst detection level and misses many targets. In general, as Figure 14 shows, if $\alpha$ decreases, the processor has a better estimation level.

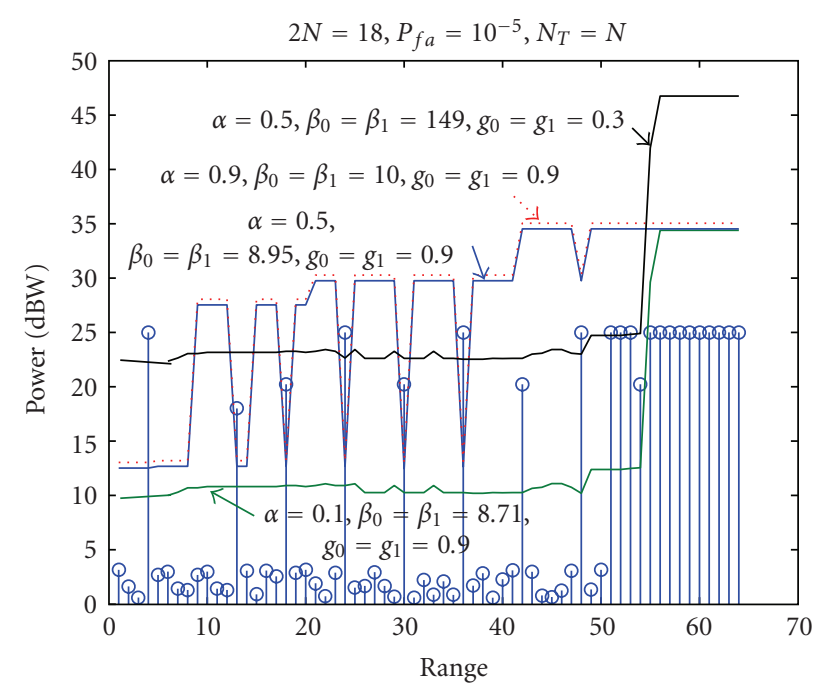

FIGURE 14: Detection thresholds of SOS I with different parameters in worst case for $2 N=18$ and $P_{f a}=10^{-5}$.

Considering the result of this section, we see that selecting larger $\beta_{0}=\beta_{1}$ and $g_{0}=g_{1}$ versus smaller $\alpha$ and $N_{T}=N$ causes less $P_{f a}$ in a homogeneous environment and better performance for $P_{d}$ in homogeneous and nonhomogeneous environments, but results in worse $P_{f a}$ in the presence of clutter edge. Therefore, to have the best performance in various radar environments, suitable parameters, as discussed, should be selected to achieve optimal performance. Also, as the results above and equations in Section 3 show, increasing $N_{T}$ causes the performance of SOS I to become similar to OS; since, based on (17) and (18), in this case all the samples in the reference window are chosen for background noise estimation (which is based on an Ordered Statistic process), therefore its performance will be near to OS.

\section{Conclusions}

Considering the results of Section 4 and the comparison with other mean-level processors, the SOS CFAR I processor has a better performance in different radar environments. Also these results show that the SOS CFAR I processor has a good performance with less detection loss not only in homogeneous environments but also in nonhomogeneous ones such as multiple targets and especially in clutter edge. In addition, simulation results confirm that the acquired detection threshold of SOS CFAR I will be optimised if the number of interfering targets is less than the size of the reference window, and it will be a processor which can detect all the targets. However, implementation of the SOS CFAR I is complex compared to conventional processors. Also, these results show that by adjusting the SOS CFAR I parameters, it has better performance than OS in the presence of clutter region and multiple targets and better performance than SCFAR in the presence of multiple targets with INR more than SNR. It means with using ordered statistic method in S-CFAR, SOS CFAR I will have better performance in the presence of multiple targets environment. 


\section{Appendices}

A.

By using (11), $P_{S}$ in (10) can be calculated as follows:

$$
\begin{aligned}
P_{S} & =E_{X_{0}}\left\{\left(\begin{array}{c}
2 N \\
n_{0}
\end{array}\right) P_{0}^{n_{0}}\left(x_{0}\right)\left(1-P_{0}\left(x_{0}\right)\right)^{2 N-n_{0}}\right\} \\
& =\int_{x_{0}=0}^{\infty}\left(\begin{array}{c}
2 N \\
n_{0}
\end{array}\right) P_{0}^{n_{0}}\left(x_{0}\right)\left(1-P_{0}\left(x_{0}\right)\right)^{2 N-n_{0}} f_{X_{0}}\left(x_{0}\right) d x_{0} \\
& =\int_{x_{0}=0}^{\infty}\left(\begin{array}{c}
2 N \\
n_{0}
\end{array}\right)\left(1-e^{-(\alpha / \lambda) x_{0}}\right)^{n_{0}}\left(e^{-(\alpha / \lambda) x_{0}}\right)^{2 N-n_{0}} \frac{1}{\lambda_{S}} e^{-x_{0} / \lambda_{S}} d x_{0} \\
& =\frac{1}{\lambda_{S}}\left(\begin{array}{c}
2 N \\
n_{0}
\end{array}\right) \int_{x_{0}=0}^{\infty} e^{-\left((\alpha / \lambda)\left(2 N-n_{0}\right)+1 / \lambda_{S}\right) x_{0}} \times \sum_{i=0}^{n_{0}}\left(\begin{array}{c}
n_{0} \\
i
\end{array}\right)\left(-e^{-(\alpha / \lambda) x_{0}}\right)^{i} d x_{0} \\
& =\frac{1}{\lambda_{S}}\left(\begin{array}{c}
2 N \\
n_{0}
\end{array}\right) \sum_{i=0}^{n_{0}}\left(\begin{array}{c}
n_{0} \\
i
\end{array}\right)(-1)^{i} \int_{x_{0}=0}^{\infty} e^{-\left((\alpha / \lambda)\left(2 N-n_{0}+i\right)+1 / \lambda_{S}\right) x_{0}} d x_{0} \\
& =\frac{1}{\lambda_{S}}\left(\begin{array}{c}
2 N \\
n_{0}
\end{array}\right) \sum_{i=0}^{n_{0}}\left(\begin{array}{c}
n_{0} \\
i
\end{array}\right)(-1)^{i} \frac{(-1)^{i}}{(\alpha / \lambda)\left(2 N-n_{0}+i\right)+1 / \lambda_{S}} \\
& =\left(\begin{array}{c}
2 N \\
n_{0}
\end{array}\right) \sum_{i=0}^{n_{0}}\left(\begin{array}{c}
n_{0} \\
i
\end{array}\right) \frac{1}{\alpha\left(2 N-n_{0}+i\right)\left(1+\sigma_{s}\right)+1} . \\
&
\end{aligned}
$$

B.

By employing (12), for calculating $P\left(X_{0}>\beta_{1} Z_{1} \mid H_{1}\right)$, one has [8]

$$
\begin{aligned}
P\left(x_{0}>\right. & \left.\beta_{1} Z_{1} \mid H_{1}\right) \\
& =\int_{z_{1}=0}^{\infty} \int_{x_{0}=\beta_{1} Z_{1}}^{\infty} \frac{1}{\lambda\left(1+\sigma_{s}\right)} e^{-x_{0} / \lambda\left(1+\sigma_{s}\right)} d x_{0} f_{K_{1}}\left(z_{1}\right) d z_{1} \\
& =\int_{z_{1}=0}^{\infty} e^{-\left(\beta_{1} / \lambda\left(1+\sigma_{s}\right)\right) z_{1}} f_{K_{1}}\left(z_{1}\right) d z_{1} \\
& =M_{z_{1}}\left(\frac{\beta_{1}}{\lambda\left(1+\sigma_{s}\right)}\right),
\end{aligned}
$$

where $M_{Z_{1}}(u)$ is the moment generating function of $Z_{1}$ and gives

$$
\begin{array}{r}
M_{Z_{1}}(u)=\int_{z_{1}=0}^{\infty} e^{-u z_{1}} K_{1}\left(\begin{array}{c}
2 N \\
K_{1}
\end{array}\right)\left(e^{-z_{1} / \lambda}\right)^{2 N-K_{1}} \\
\quad \times\left(1-e^{-z_{1} / \lambda}\right)^{K_{1}-1} \frac{1}{\lambda} e^{-z_{1} / \lambda} d z \\
=K_{1}\left(\begin{array}{c}
2 N \\
K_{1}
\end{array}\right) \frac{1}{\lambda} \int_{z_{1}=0}^{\infty}\left(1-e^{-z_{1} / \lambda}\right)^{K_{1}-1} \\
\times e^{-\left(u+\left(2 N-K_{1}+1\right) / \lambda\right) z_{1}} d z_{1}
\end{array}
$$

$$
\begin{array}{r}
=K_{1}\left(\begin{array}{c}
2 N \\
K_{1}
\end{array}\right) \frac{1}{\lambda} \int_{z_{1}=0}^{\infty} \sum_{m=0}^{K_{1}-1}\left(\begin{array}{c}
K_{1}-1 \\
m
\end{array}\right)\left(-e^{-z_{1} / \lambda}\right)^{m} \\
\times e^{-\left(u+\left(2 N-K_{1}+1\right) / \lambda\right) z_{1}} d z_{1}
\end{array}
$$$$
=K_{1}\left(\begin{array}{c}
2 N \\
K_{1}
\end{array}\right) \frac{1}{\lambda} \sum_{m=0}^{K_{1}-1}\left(\begin{array}{c}
K_{1}-1 \\
m
\end{array}\right)(-1)^{m}
$$$$
\times \int_{z_{1}=0}^{\infty} e^{-\left(u+\left(2 N-K_{1}+1\right) / \lambda+m / \lambda\right) z_{1}} d z_{1}
$$$$
=K_{1}\left(\begin{array}{c}
2 N \\
K_{1}
\end{array}\right) \frac{1}{\lambda} \sum_{m=0}^{K_{1}-1}\left(\begin{array}{c}
K_{1}-1 \\
m
\end{array}\right)(-1)^{m}
$$$$
\times \frac{1}{u+\left(2 N-K_{1}+1\right) / \lambda+m / \lambda} .
$$

Therefore by setting $u=\beta_{1} / \lambda\left(1+\sigma_{s}\right)$ in (B.2),

$$
\begin{aligned}
P\left(X_{0}>\right. & \left.\beta_{1} Z_{1} \mid H_{1}\right) \\
= & \frac{(2 N) !}{\left(2 N-K_{1}\right) !} \\
& \times \sum_{m=0}^{K_{1}-1} \frac{(-1)^{m}}{m !\left(K_{1}-m-1\right) !} \frac{1}{\beta_{1} /\left(1+\sigma_{s}\right)+2 N-K_{1}+1+m} .
\end{aligned}
$$

\section{C.}

Referring to (11) and (22), $Q_{S}$ in (21) can be calculated in the following manner:

$$
\begin{aligned}
& Q_{s}=E_{X_{0}}\left\{\sum_{m=m_{1}}^{\min \left(M, n_{0}\right)}\left(\begin{array}{c}
2 N-M \\
n_{0}-m
\end{array}\right)\left(\begin{array}{l}
M \\
m
\end{array}\right)\left(P_{0}\left(X_{0}\right)\right)^{n_{0}-m}\right. \\
& \times\left(1-P_{0}\left(X_{0}\right)\right)^{2 N-M-\left(n_{0}-m\right)} \\
& \left.\times\left(P_{0}^{\prime}\left(X_{0}\right)\right)^{n_{0}-m}\left(1-P_{0}^{\prime}\left(X_{0}\right)\right)^{M-m}\right\} \\
& =\int_{X_{0}=0}^{\infty} \sum_{m=m_{1}}^{\min \left(M, n_{0}\right)}\left(\begin{array}{c}
2 N-M \\
n_{0}-m
\end{array}\right)\left(\begin{array}{l}
M \\
m
\end{array}\right)\left(P_{0}\left(X_{0}\right)\right)^{n_{0}-m} \\
& \times\left(1-P_{0}\left(X_{0}\right)\right)^{2 N-M-\left(n_{0}-m\right)} \\
& \times\left(P_{0}^{\prime}\left(X_{0}\right)\right)^{m}\left(1-P_{0}^{\prime}\left(X_{0}\right)\right)^{M-m} f_{X_{0}}\left(x_{0}\right) d x_{0} \\
& =\sum_{m=m_{1}}^{\min \left(M, n_{0}\right)}\left(\begin{array}{c}
2 N-M \\
n_{0}-m
\end{array}\right)\left(\begin{array}{l}
M \\
m
\end{array}\right) \\
& \times \int_{x_{0}=0}^{\infty}\left(1-e^{-(\alpha / \lambda) x_{0}}\right)^{n_{0}-m}\left(e^{-(\alpha / \lambda) x_{0}}\right)^{2 N-M-\left(n_{0}-m\right)} \\
& \times\left(1-v e^{-\left(\alpha / \lambda_{I}\right) x_{0}}\right)^{m}\left(e^{-\left(\alpha / \lambda_{I}\right) x_{0}}\right)^{M-m} \frac{1}{\lambda_{S}} e^{-x_{0} / \lambda_{S}} d x_{0}
\end{aligned}
$$




$$
\begin{aligned}
& =\sum_{m=m_{1}}^{\min \left(M, n_{0}\right)}\left(\begin{array}{c}
2 N-M \\
n_{0}-m
\end{array}\right)\left(\begin{array}{l}
M \\
m
\end{array}\right) \\
& \times \int_{x_{0}=0}^{\infty} \frac{1}{\lambda_{S}} e^{-\left(\left(\left(2 N-M-\left(n_{0}-m\right)\right) / \lambda\right) \alpha+\left((M-m) / \lambda_{I}\right) \alpha+1 / \lambda_{S}\right) x_{0}} \\
& \times \sum_{t=0}^{n_{0}-m}\left(\begin{array}{c}
n_{0}-m \\
t
\end{array}\right)(-1)^{t} e^{-((\alpha / \lambda) t) x_{0}} \\
& \times \sum_{q=0}^{m}\left(\begin{array}{c}
m \\
q
\end{array}\right)(-1)^{q} e^{-\left(\left(\alpha / \lambda_{I}\right) q\right) x_{0}} d x_{0} \\
& =\sum_{m=m_{1}}^{\min \left(M, n_{0}\right)}\left(\begin{array}{c}
2 N-M \\
n_{0}-m
\end{array}\right)\left(\begin{array}{c}
M \\
m
\end{array}\right) \\
& \times \frac{1}{\lambda_{S}} \sum_{t=0}^{n_{0}-m} \sum_{q=0}^{m}\left(\begin{array}{c}
n_{0}-m \\
t
\end{array}\right)\left(\begin{array}{c}
m \\
q
\end{array}\right)(-1)^{t+q} \\
& \times \int_{x_{0}=0}^{\infty} e^{-\left(\left(\left(2 N-M-\left(n_{0}-m\right)\right) / \lambda\right) \alpha+\left((M-m) / \lambda_{I}\right) \alpha+1 / \lambda_{S}+(t / \lambda) \alpha+\left(q / \lambda_{I}\right) \alpha\right) x_{0}} d x_{0} \\
& =\sum_{m=m_{1}}^{\min \left(M, n_{0}\right)}\left(\begin{array}{c}
2 N-M \\
n_{0}-m
\end{array}\right)\left(\begin{array}{l}
M \\
m
\end{array}\right) \\
& \times \sum_{t=0}^{n_{0}-m} \sum_{q=0}^{m} \frac{(-1)^{t+q}\left(\begin{array}{c}
n_{0}-m \\
t
\end{array}\right)\left(\begin{array}{c}
m \\
q
\end{array}\right)}{1+\mathfrak{N}\left(1+\sigma_{s}\right) \alpha+(M-m+q)\left(\left(1+\sigma_{s}\right) /\left(1+\sigma_{I}\right)\right) \alpha} .
\end{aligned}
$$

\section{D.}

For calculating $P\left(X_{0}>\beta_{1} Z_{1} \mid H_{1}\right)$, one has

$$
\begin{aligned}
P\left(X_{0}\right. & \left.>\beta_{1} Z_{1} \mid H_{1}\right) \\
& =\int_{z_{1}=0}^{\infty} \int_{x_{0}=\beta_{1} Z_{1}}^{\infty} \frac{1}{\lambda\left(1+\sigma_{s}\right)} e^{-x_{0} / \lambda\left(1+\sigma_{s}\right)} d x_{0} f_{K_{1}}\left(z_{1}\right) d z_{1} \\
& =\int_{z_{1}=0}^{\infty} e^{-\left(\beta_{1} / \lambda\left(1+\sigma_{s}\right)\right) z_{1}} f_{K_{1}}\left(z_{1}\right) d z_{1} \\
& =\frac{\beta_{1}}{\lambda\left(1+\sigma_{s}\right)} \int_{z_{1}=0}^{\infty} F_{K_{1}}\left(z_{1}\right) e^{-\left(\beta_{1} / \lambda\left(1+\sigma_{s}\right)\right) z_{1}} d z_{1} .
\end{aligned}
$$

Here, $F_{k 1}\left(z_{1}\right)$ is the CDF of $f_{k 1}\left(z_{1}\right)$ in the case of $M$ interfering samples in the reference window and is equal to [10]

$$
\begin{aligned}
F_{K_{1}}\left(z_{1}\right)=\sum_{i=k_{1}} \sum_{L=\max (0, i-M)}^{\min (i, 2 N-M)}\left(\begin{array}{c}
2 N-M \\
L
\end{array}\right)\left(\begin{array}{c}
M \\
i-L
\end{array}\right) \\
\times e^{-(2 N-M-L) z_{1}}\left(1-e^{\left.-z_{1}\right)^{L}}\right. \\
\times e^{-(M-i+L)\left(z_{1} /\left(1+\sigma_{I}\right)\right)}\left(1-e^{-z_{1} /\left(1+\sigma_{I}\right)}\right)^{i-L} .
\end{aligned}
$$

Therefore, (D.1) will be

$$
\begin{aligned}
P\left(X_{0}>\beta_{1} Z_{1} \mid H_{1}\right) \\
=\frac{\beta_{1}}{\left(1+\sigma_{s}\right)} \sum_{i=k_{1}}^{2 N} \sum_{L=p_{1}}^{p_{2}}\left(\begin{array}{c}
2 N-M \\
L
\end{array}\right)\left(\begin{array}{c}
M \\
i-L
\end{array}\right) \\
\quad \times \sum_{j_{1}=0}^{L} \sum_{j_{2}=0}^{i-L} \frac{\left(\begin{array}{c}
L \\
j_{1}
\end{array}\right)\left(\begin{array}{c}
i-L \\
j_{2}
\end{array}\right)(-1)^{j_{1}+j_{2}}}{2 N-M-L+\beta_{1} /\left(1+\sigma_{s}\right)+j_{1}+\mathfrak{Q} /\left(1+\sigma_{I}\right)},
\end{aligned}
$$

where $p_{1}$ and $p_{2}$ are $\max (0, i-M)$ and $\min (i, 2 N-M)$.

\section{References}

[1] H. Rohling, "Some radar topics: waveform design, range CFAR and target recognition," in Advances in Sensing with Security Applications, vol. 2 of NATO Security through Science Series, pp. 293-322, Springer, Amsterdam, The Netherlands, 2006.

[2] Y. I. Han and T. Kim, "Performance of excision GO-CFAR detectors in nonhomogeneous environments," IEE Proceedings: Radar, Sonar and Navigation, vol. 143, no. 2, pp. 105-111, 1996.

[3] H. Goldman, "Performance of the excision CFAR detector in the presence of interferers," IEE Proceedings, Part F: Radar and Signal Processing, vol. 137, no. 3, pp. 163-171, 1990.

[4] S. Erfanian and V. T. Vakili, "Analysis of improved switching CFAR in the presence of clutter and multiple targets," in Proceedings of the 50th International Symposium ELMAR-2008, vol. 1, pp. 257-260, Zadar, Croatia, September 2008.

[5] S. Erfanian and V. T. Vakili, "Optimum detection of multiple targets by improved switching CFAR processor," in Proceedings of the 14th Asia-Pacific Conference on Communications (APCC '08), pp. 1-5, Tokyo, Japan, October 2008.

[6] M. Barkat, Signal Detection and Estimation, Artech House, Boston, Mass, USA, 2005.

[7] S. Erfanian and S. Faramarzi, "Performance of excision switching-CFAR in $\mathrm{K}$ distributed sea clutter," in Proceedings of the 14th Asia-Pacific Conference on Communications (APCC '08), pp. 1-4, Tokyo, Japan, October 2008.

[8] T.-T. Van Cao, "A CFAR thresholding approach based on test cell statistics," in Proceedings of IEEE National Radar Conference, pp. 349-354, Philadelphia, Pa, USA, April 2004.

[9] T.-T. Van Cao, "A CFAR algorithm for radar detection under severe interference," in Proceedings of the Intelligent Sensors, Sensor Networks and Information Processing Conference (ISSNIP '04), pp. 167-172, Melbourne, Canada, December 2004.

[10] P. P. Gandhi and S. A. Kassam, "Analysis of CFAR processors in homogeneous background," IEEE Transactions on Aerospace and Electronic Systems, vol. 24, no. 4, pp. 427-445, 1988.

[11] R. Peihong, D. Qingfen, and C. Yuanhen, "The research on the detection performance of OS-CFAR and its modified methods," in Proceedings of CIE International Conference of Radar (ICR '96), pp. 422-425, Beijing, China, October 1996.

[12] H. Rohling, "Radar CFAR thresholding in clutter and multiple target situations," IEEE Transactions on Aerospace and Electronic Systems, vol. 19, no. 4, pp. 608-621, 1983.

[13] V. G. Hansen and J. H. Sawyers, "Detectability loss due to "greatest of" selection in a cell-averaging CFAR," IEEE Transactions on Aerospace and Electronic Systems, vol. 16, no. 1, pp. 115-118, 1980. 